\title{
Desenvolvimento do Instrumento de Avaliação Neuropsicológica Breve Infantil NEUPSILIN- INF
}

\author{
Jerusa Fumagalli de Salles' - Universidade Federal do Rio Grande do Sul, Porto Alegre, Brasil \\ Rochele Paz. Fonseca - Pontifícia Universidade Católica do Rio Grande do Sul, Porto Alegre, Brasil \\ Camila Cruz-Rodrigues - Universidade Federal de São Paulo e Universidade Presbiteriana Mackenzie, São Paulo, Brasil \\ Claudia B. Mello - Universidade Federal de São Paulo, São Paulo, Brasil \\ Thais Barbosa - Universidade Federal de São Paulo, São Paulo, Brasil \\ Mônica C. Miranda - Universidade Federal de São Paulo, São Paulo, Brasil
}

\begin{abstract}
Resumo
O objetivo deste estudo é apresentar o processo de desenvolvimento e validação de conteúdo do Instrumento de Avaliação Neuropsicológica Breve Infantil NEUPSILIN-INF, que avalia, de modo breve, componentes de oito funções neuropsicológicas em crianças em idade escolar: orientação, atenção, percepção visual, memória, habilidades aritméticas, linguagem, habilidades visuoconstrutivas e funções executivas. O processo envolveu: 1) análise do instrumento original NEUPSILIN e definição das funções e tarefas a serem adaptadas para avaliação neuropsicológica infantil; 2) desenvolvimento de novas tarefas consideradas fundamentais para a avaliação na infância; 3) estudo piloto 1 com a versão preliminar do instrumento; 4) análise de juízes especialistas; 5) estudos piloto 2 e 3 , nova reformulação de tarefas do instrumento e elaboração de sua versão final. $\mathrm{O}$ instrumento apresentou adequada validade aparente e de conteúdo. Palavras-chave: Criança, Testes neuropsicológicos, Neuropsicologia, Cognição, Psicometria.
\end{abstract}

\section{Development of the Child Brief Neuropsychological Assessment Battery NEUPSILIN-INF}

\begin{abstract}
The aim of this study is to present the development process and content validation of Child Brief Neuropsychological Assessment Battery NEUPSILIN-INF, which briefly assesses the components of eight neuropsychological functions in school-aged children: orientation, attention, visual perception, memory, arithmetic abilities, language, visuoconstructive abilities and executive functions. The process comprised: 1) the analysis of the original NEUPSILIN instrument and definition of the functions and tasks to be adapted for the child neuropsychological assessment; 2) the development of new tasks considered as fundamental for the assessment in children; 3) pilot study 1 with the preliminary version of the instrument; 4) analysis by specialist judges; 5) pilot studies 2 and 3, new reformulation of the instrument's tasks and preparation of its final version. The instrument presented appropriate face and content validity.

Keywords: Children, Neuropsychological tests, Neuropsychology, Cognition, Psychometrics.
\end{abstract}

A avaliação neuropsicológica infantil envolve aspectos como maturação biológica, desenvolvimento cognitivo, relação entre cognição, ensino formal, estilos de interação familiar e cultura, entre outros. O exame neuropsicológico é fundamental para auxiliar na definição de quadros clínicos na infância, como a dislexia ou o transtorno do déficit de atenção/hiperatividade. Cabe ao profissional, não só estabelecer o perfil dos déficits (fragilidades) e sua extensão funcional, mas também as habilidades preservadas (potencialidades) (Miranda, 2006). Nessa avaliação não é tão importante estabelecer relações anatomoclínicas, tendo em vista a acentuada variabilidade entre substrato neuroanatômico e manifestações comportamentais em crianças, por estarem em desenvolvimento neural (Simões, 2002).

Em face dessas particularidades da infância, devem ser adotados paradigmas clínicos de avaliação

\footnotetext{
${ }^{1}$ Endereço para correspondência:

Instituto de Psicologia, UFRGS, Avenida Ipiranga, 2.600, sala 114. 90035-003 - Porto Alegre-RS. Brasil.

E-mail: jerusafsalles@gmail.com
}

cognitiva desenvolvidos especificamente para esta população. São usados procedimentos como anamnese (direcionada a familiares/responsáveis da criança), observações clínicas, análise de exames complementares, administração de tarefas ecológicas, aplicação de escalas e testes neuropsicológicos padronizados (Strauss, Sherman \& Spreen, 2006). Estes últimos diferenciam-se em procedimentos de rastreio (screening), instrumentos de exame breve e baterias completas.

A avaliação pode iniciar com a identificação de possíveis alterações do desenvolvimento neuropsicológico por meio de um instrumento de aplicação mais rápida (Lezak, Howieson \& Loring, 2004). As crianças identificadas como apresentando déficits são submetidas a procedimentos de avaliação com baterias mais completas, aprofundando-se a avaliação dos processos cognitivos de interesse. Reitan e Wolfson (2004a) salientam que o objetivo da avaliação é proporcionar informações que podem auxiliar no delineamento de estratégias apropriadas de intervenção, orientadas para promover o desenvolvimento das funções neuropsicológicas ou 
para minimizar o impacto de disfunções sobre a aprendizagem e o comportamento.

É importante considerar, como destacam Carey e Konkol (2001), que a viabilidade da avaliação neuropsicológica tradicional em serviços públicos de assistência à infância vem sendo questionada por administradores da área da saúde. Uma avaliação neuropsicológica infantil adequada aos propósitos de intervenção e economicamente viável tem sido um desafio para os centros de saúde no Brasil. Além disso, sabe-se da limitação que a restrita quantidade de instrumentos de avaliação em nosso país impõe ao exame neuropsicológico como um todo e, em especial, na faixa de desenvolvimento infantil.

Em face desta grande demanda existente de instrumentos específicos para avaliação cognitiva infantil, o desenvolvimento ou adaptação de novos instrumentos para crianças é necessário, incluindo processos de adaptação (ou construção) para diferentes culturas (Byrd, Arentoft, Scheiner, Westerveld \& Baron, 2008; Hambleton \& Bollwark, 1991). No que tange aos instrumentos de avaliação neuropsicológica infantil, há três práticas comuns observadas na literatura: 1) uso de instrumento desenvolvido para todo o ciclo vital com normas para crianças (como exemplo o Teste Wisconsin de Classificação de Cartas), 2) desenvolvimento de instrumentos específicos para crianças (tal como a Children's Memory Scale, desenvolvida por Cohen em 1997), e 3) adaptação de instrumentos elaborados para adultos em busca de uma versão aplicável e sensível para crianças (exemplo: versão Teddy Bear Cancellation Test de Laurent-Vannier, Chevignard, Pradat-Diehl, Abada \& De Agostini, 2006).

O presente estudo tem por objetivo apresentar o processo de desenvolvimento e validação de conteúdo do Instrumento de Avaliação Neuropsicológica Breve Infantil NEUPSILIN-INF (Salles, Fonseca, Miranda, Mello, Cruz-Rodrigues \& Barbosa, no prelo). Este processo tomou como base algumas tarefas do Instrumento de Avaliação Neuropsicológica Breve NEUPSILIN (Fonseca, Salles \& Parente, 2008, 2009). O teste visa a fornecer um perfil breve do funcionamento de processos neuropsicológicos (preservados ou desenvolvidos, e deficitários), de caráter quantitativo e qualitativo.

\section{Método}

O processo de desenvolvimento do NEUPSILIN-INF seguiu 5 etapas sucessivas recomendadas pela literatura internacional (Hogan, 2006), descritas a seguir:
Etapa 1 - Análise do instrumento original NEUPSILIN e definição das funções e tarefas a serem adaptadas para avaliação neuropsicológica infantil: a partir da experiência e da validade do NEUPSILIN (Fonseca, Salles \& Parente, 2009), as funções, tarefas e processos foram analisados e aqueles aplicáveis na infância foram selecionados. Estas provas foram adaptadas, ou seja, providas com instruções e estímulos apropriados (nível linguístico, atencional e mnemônico) para a faixa de desenvolvimento de crianças em idade escolar.

Etapa 2 - Desenvolvimento de novas tarefas consideradas fundamentais para a avaliação na infância: consultou-se sistematicamente a literatura concernente aos modelos teóricos e aos instrumentos e tarefas de avaliação de funções neuropsicológicas (incluindo avaliação de linguagem) de uso frequente na prática clínica na infância (Gathercole, 1998; Lezak e colaboradores, 2004; Matute, Rosselli, Ardila \& Ostrosky-Solis, 2007; Salles \& Parente, 2008). Esta etapa foi importante para definir os atributos ou propriedades das funções a serem estudadas, suas definições conceituais e operacionais, gerando a versão preliminar do instrumento.

\section{Etapa 3 - Estudo piloto 1}

A primeira versão do NEUPSILIN-INF foi aplicada em 29 crianças (16 provenientes do Rio Grande do Sul e 13 de São Paulo), representando os futuros grupos normativos, categorizados por: a) idade/série, 6 anos $(\mathrm{n}=3), 7,8$ e 9 anos $(\mathrm{n}=4 \mathrm{em}$ cada grupo), 10 anos ( $n=5), 11$ anos $(n=7)$ e 12 anos de idade ( $n=2)$; b) tipo de escola (12 de escola pública e 17 de escola privada); c) sexo (15 meninas e 14 meninos). A aplicação foi individual, seguindo os procedimentos de aplicação e registro dessa primeira versão do manual do instrumento. Para a análise dos dados, as autoras se reuniram com os aplicadores para discutir as dificuldades encontradas em cada item quanto à validade técnica, visando: a) testar o instrumento em uma situação real de coleta para verificar possíveis falhas, tais como uso de termos não compreensíveis aos participantes e ambiguidade de alguma instrução, entre outras; b) estimar a duração da aplicação; c) adequar as tarefas, estímulos e instruções à amostraalvo; e d) analisar o valor funcional do instrumento (se atingiu o efeito teto em alguma faixa etária, a variabilidade dos escores entre crianças de diferentes idades, entre outros). Com base nessa análise e discussão, os estímulos e normas de instrução, interpretação e pontuação foram reformulados, obtendo-se a segunda versão do NEUPSILIN-INF.

Etapa 4 - Análise de juízes especialistas 
A análise dos itens e das instruções foi feita por nove juízes especialistas brasileiros, pesquisadores da área de desenvolvimento infantil, sendo seis com experiência em neuropsicologia, dois em avaliações de linguagem e um perito em avaliação psicológica. Esse procedimento foi realizado em duas fases: a) julgamento de qual função ou componente cognitivo/neuropsicológico está sendo predominantemente examinado(a) no conjunto de instrução-estímulo(s), e b) análise da importância da inclusão de cada subteste, considerando o construto a ser avaliado e o objetivo do instrumento. O juiz deveria apontar se o item/função era necessário ou dispensável e fazer sugestões que julgasse pertinentes. Os autores discutiram a análise de todos os juízes, reformularam o instrumento, gerando assim a terceira versão do NEUPSILIN-INF. Calculou-se um índice simples de concordância, com base no método de Fagundes (1985), ou seja, número de concordâncias dividido pelo número total de itens das tarefas de análise de juízes especialistas.

Os novos estímulos-itens, após a reformulação proposta pelos juízes, foram analisados ainda por dois outros juízes especialistas em neuropsicologia da memória. As críticas e sugestões foram submetidas a um consenso efetuado pelos autores do instrumento, gerando a quarta versão do instrumento NEUPSILININF.

\section{Etapa 5 - Estudos piloto 2 e 3 e elaboração da versão final do instrumento}

O segundo estudo piloto contou com 56 crianças, com idade média de 10,3 anos $(D P=0,43)$, sendo 30 meninos $(53,6 \%)$, estudantes da rede pública de $2^{\mathrm{a}} \quad\left(3^{\circ}\right.$ ano) a $5^{\mathrm{a}} \quad\left(6^{\circ}\right.$ ano $)$ séries do ensino fundamental de escolas de Porto Alegre-RS (para detalhamento ver Piccolo, 2010). Com os mesmos objetivos do estudo piloto 1, este estudo testou a quarta versão do instrumento NEUPSILIN-INF. A partir dele foram feitas modificações, especialmente relativas às instruções das tarefas, com vistas a favorecer a compreensão por parte dos integrantes da amostra, assim como adequação dos materiais de aplicação (protocolo de registro) para facilitar o trabalho dos avaliadores.

O terceiro e último estudo piloto, realizado a partir de julho de 2009, com a quinta e atual versão do instrumento, contou com a participação de mais 14 crianças, uma de cada idade, entre 6 e 12 anos, do $1^{\circ}$ até o $6^{\circ}$ ano do ensino fundamental, procedentes dos estados do RS e de SP. Foi realizada a discussão com a equipe de coleta de dados, como descrito no primeiro estudo piloto, tendo o foco maior nos critérios de pontuação, como escores de acertos, categorização de erros e estratégias de resolução adotadas pelas crianças em cada subteste (análises quanti e qualitativas).

Ressalta-se que, para a análise semântica dos itens, em cada estudo piloto, os participantes dos grupos de coleta de dados (estudantes e profissionais da área da saúde) foram entrevistados na modalidade brainstorming. Os itens com dificuldades de compreensão pelos participantes foram substituídos e reapresentados. Este procedimento repetiu-se em várias etapas do processo de desenvolvimento do NEUPSILIN-INF, tendo sido de especial importância para as instruções de aplicação e normas de pontuação do instrumento.

\section{Resultados}

\section{NEUPSILIN-INF}

O Instrumento de Avaliação Neuropsicológica Breve Infantil NEUPSILIN-INF examina o desempenho em oito funções neuropsicológicas, por meio de 26 subtestes: orientação, atenção focalizada, percepção visual e de emoções em faces, memória verbal e visual (de trabalho ou operacional, episódica e semântica), habilidades aritméticas, linguagem oral, leitura e escrita, habilidades visuocontrutivas e funções executivas. Inicialmente, a seleção dessas oito funções foi feita com base na literatura acerca da neuropsicologia do desenvolvimento e no estudo de outros instrumentos neuropsicológicos infantis, e ainda tendo análise de sua relevância por juízes especialistas, como já descrito nas etapas 2 e 4 . Sua versão final resultou em tarefas curtas e de resolução acessível a crianças na faixa de idade de 6 a 12 anos. Pode ser classificado como um instrumento de avaliação neuropsicológica breve por possuir um tempo reduzido de aplicação (entre 40 e 60 minutos, dependendo da faixa etária da criança) e promover uma avaliação mais ampla do que um simples screening. A Tabela 1 apresenta uma descrição sintética da aplicação e estímulos para cada tarefa. 
Tabela 1. Tarefa/função, aplicação e itens da versão final do Instrumento de Avaliação Neuropsicológica Breve Infantil NEUPSILIN-INF

\begin{tabular}{|c|c|c|}
\hline Tarefa & $\begin{array}{l}\text { Tipo de aplicação } \\
\text { (apresentação) }\end{array}$ & Descrição do item \\
\hline 1. Orientação & oral & 6 questões \\
\hline 2. $\quad$ Atenção & & \\
\hline $\begin{array}{l}2.1 \text { Atenção visual - Cancelamento de } \\
\text { figuras }\end{array}$ & visual & 202 figuras, 35 alvos \\
\hline 2.2 Atenção auditiva - sequência de dígitos & oral & Repetição oral de números -2 a 5 itens \\
\hline 3. Percepção & & \\
\hline $\begin{array}{l}\text { 3.1 Percepção de emoção em faces } \\
\text { 3.2 Percepção visual - constância de forma e } \\
\text { constância do objeto }\end{array}$ & $\begin{array}{l}\text { visual } \\
\text { visual }\end{array}$ & $\begin{array}{l}6 \text { imagens de faces } \\
\text { O alvo é comparado a duas figuras }\end{array}$ \\
\hline
\end{tabular}

4. Memória

4.1 Memória de trabalho (operacional)

4.1.1 Sequência de dígitos na ordem indireta oral

4.1.2 Span de pseudopalavras

oral

4.1.3 Memória de trabalho (operacional)

visoespacial (ordem inversa)

4.2 Memória episódicosemântica

4.2.1 Recordação imediata e tardia de

palavras

4.2.2 Recordação imediata de figuras

4.2.3 Memória semântica

oral
visual - página com 8
quadrados

oral

visual - páginas separadas oral

\section{Linguagem}

5.1 Linguagem oral

5.1.1 Nomeação

5.1.2 Consciência fonológica

i) Rima

ii) Subtração fonêmica

5.1.3 Compreensão oral

5.1.4 Processamento inferencial

5.2 Linguagem escrita

5.2.1 Leitura em voz alta

i) Sílabas

ii) Palavras

ii) Pseudopalavras

5.2.2 Compreensão escrita

5.2.3 Escrita de palavras e de pseudopalavras

5.2.4 Escrita espontânea

5.2.5 Escrita copiada

6. Habilidades visoconstrutivas

6.1 Cópia de figuras

7. Habilidades aritméticas

7.1 Contagem de palitos

7.2 Cálculos matemáticos

8. Funções executivas

8.1 Fluência verbal

i) Ortográfica

ii) Semântica

8.2 Tarefa go-no go auditiva visual

oral

oral

visual -3 páginas
oral

visual- 6 páginas

visual- 6 páginas

visual- 5 páginas

visual- 5 páginas

oral

oral

visual -1 página

visual -4 páginas

visual - 1 página

oral

oral
oral
oral - gravada

Repetição oral de dígitos -2 a 5 itens Repetição oral de pseudopalavras -1 a 4 itens Apontar a sequência de 2 a 5 quadrados

Recordação oral de 9 palavras

Recordação oral de 9 figuras 4 questões 


\section{Resultados das etapas 1 e 2}

Julgaram-se como importantes e potencialmente sensíveis para a avaliação neuropsicológica infantil as seguintes funções e tarefas do NEUPSILIN (Fonseca e colaboradores, 2009): orientação; atenção auditiva - repetição de sequência de dígitos (ordem direta); memória episódico-semântica verbal (evocação imediata - palavras), memória semântica; linguagem oral - nomeação, compreensão oral, processamento inferencial; linguagem escrita leitura em vOz alta de palavras e pseudopalavras, compreensão escrita, escrita de palavras e de pseudopalavras, escrita espontânea, escrita copiada, habilidades viso-construtivas - cópia de figuras; habilidades aritméticas - cálculos matemáticos; funções executivas - fluência verbal ortográfica.

Os estímulos das tarefas do NEUPSILIN (Fonseca e colaboradores, 2009) constituídos por imagens (como em compreensão oral e escrita) foram modificados e confeccionados de forma a serem atrativos para crianças (coloridos, com traços infantis). A maioria dos estímulos e instruções do instrumento original sofreu algum ajuste. Por exemplo, os itens que compõem a tarefa de memória episódico-semântica foram modificados a fim de conter estímulos adequados à infância, a partir da tarefa desenvolvida no estudo do desenvolvimento da memória de Miranda (2000). Em relação à atenção focalizada, o subteste contagem inversa do NEUPSILIN foi substituído pela tarefa Cancelamento de Figuras. Foram mantidos alguns itens das tarefas: orientação; span ascendente de dígitos - memória de trabalho ou operacional (que foi substituída pela repetição de dígitos na ordem indireta); compreensão oral; cópia de figuras; e habilidades aritméticas.

Considerando a faixa etária a ser estudada e o objetivo do instrumento de auxiliar na detecção de possíveis transtornos de desenvolvimento, foram construídas/adaptadas tarefas de: 1) processamento fonológico da linguagem, entre elas consciência fonológica (uma habilidade metalinguística) e memória de trabalho (operacional) fonológica (repetição de pseudopalavras); 2) processamento de emoções em faces; 3) funções executivas, componente de inibição (tarefa go-no go); 4) memória de trabalho, componente visuoespacial (semelhante aos blocos de Corsi, Lezak e colaboradores, 2004); 5) leitura de sílabas (complementando a leitura de palavras e pseudopalavras); entre outras. Ao todo, construíram-se os seguintes subtestes: atenção visual - cancelamento de figuras; percepção de emoção em faces; percepção visual - constância de forma e constância do objeto; memória de trabalho (operacional) - repetição de sequência de dígitos na ordem inversa e span de pseudopalavras; memória de trabalho (operacional) visoespacial (ordem inversa); memória episódicosemântica visoverbal (evocação imediata - figuras); linguagem oral - consciência fonológica (rima e subtração fonêmica); leitura em voz alta de sílabas; habilidades aritméticas - contagem de palitos (considerando as crianças de 6 anos); funções executivas - fluência verbal semântica e tarefa go-no go auditiva.

Embora a tarefa de span de dígitos na ordem direta possa ser concebida tradicionalmente como memória de curto prazo, também demanda atenção focalizada de estímulos verbais (componente fonológico da memoria de trabalho envolvido). A tarefa de span de pseudopalavras é considerada tarefa clássica para avaliar o componente fonológico da memória de trabalho, como uma tarefa simples de span (ver Alloway, Gathercole, Willis \& Adams, 2004).

\section{Resultados da Etapa 3 - Estudo piloto 1}

O estudo mostrou que o tempo médio de aplicação do instrumento foi de 46 minutos (DP= 13,0) e houve tendência de escores mais elevados conforme aumento da idade/série na maioria das funções neuropsicológicas avaliadas. A tarefa de percepção, que inicialmente tinha três itens, apresentou efeito teto e, portanto, foi modificada para ter seis itens. A partir da idade de nove anos, as provas associadas às funções de memória semântica, habilidades aritméticas e orientação mostraram efeito teto, mas optou-se por mantê-las devido à possibilidade de detecção precoce de déficits.

As tarefas de atenção (cancelamento de figuras) e de funções executivas (tarefa go-no go) sofreram alterações, pois o estudo piloto mostrou que elas eram pouco sensíveis. O cancelamento de figuras teve efeito teto em todas as idades, e na tarefa go-no go os escores foram bastante altos (variaram de 31 a 36, que é a pontuação máxima). Dessa forma, no cancelamento de figuras optou-se por fazer a apresentação dos estímulos (figuras nomeáveis extraídas de Pompéia, Miranda \& Bueno, 2001) de forma não-estruturada na folha de aplicação, ao contrário da primeira versão, na qual os estímulos estavam organizados por linha. Em relação à tarefa gono go, apesar dos examinadores referirem dificuldade de manipulação do caderno de aplicação (dificuldade de homogeneizar o tempo de apresentação dos estímulos), optou-se por manter essa versão para ser analisada pelos juízes quanto a sua adequação e relevância. 


\section{Resultados da Etapa 4 - análise dos juízes}

Houve concordância entre os juízes (em torno de $90 \%$ ) na primeira etapa da análise, que consistia em julgar qual função ou componente neuropsicológico estava sendo predominantemente examinado no conjunto de instrução-estímulo(s). $\mathrm{Na}$ parte 2 de análise de juízes especialistas, pertinência do conjunto instrução-estímulos para cada função/tarefa, a concordância variou de 11,1\% (1 juiz, em uma tarefa) a $100 \%$. Em relação à análise qualitativa das sugestões, apenas as tarefas de linguagem automática (contar de 1 a 10 e dizer em ordem os dias da semana) e de escrita de números foram apontadas como desnecessárias ou dispensáveis.

Após analisar as sugestões dos juízes, os autores entraram em consenso sobre quais modificações/sugestões acatar. Dessa forma, foi inserido um número maior de estímulos na tarefa de percepção (3.1) e substituída a imagem de um relógio de pulso por um relógio de cozinha na tarefa 3.2. A ordem de aplicação da tarefa de recordação de palavras também foi modificada, a fim de evitar interferência. Optou-se por excluir as tarefas de linguagem automática e incluir e substituir itens no subteste de linguagem escrita (5.2). Além disso, no teste de habilidades aritméticas foi excluída a tarefa de escrita de números, e os critérios de correção foram revisados.

Ainda, foi sugerida a inserção de tarefas de nomeação rápida (velocidade de processamento), de orientação envolvendo noções espaciais e lateralidade (D/E), e de avaliação de estereognosia e de gnosia digital. Estas sugestões, embora muito ricas para uma bateria completa expandida, não foram acatadas pelo fato de o NEUPSILIN-INF ser uma bateria breve e poder ser complementada com uso de outros instrumentos, quando o avaliador julgar necessário.

A análise final, após reformulações dos autores, foi feita por mais dois juízes especialistas, que tiveram acesso ao material integral referente a cada tarefa. As modificações foram em relação ao subteste go-no go e cancelamento de figuras. A sugestão dos juízes foi de que a tarefa go-no go fosse computadorizada, ao invés da versão visual e associada a um material que dificultava sua aplicação. Porém, como o objetivo do instrumento é ser prático e de baixo custo, acatou-se a segunda sugestão de fazer a tarefa na modalidade auditiva, como apresentado na versão final do instrumento. Os juízes também sugeriram inserir tarefas de capacidade de memória de trabalho (operacional), como, por exemplo, uma tarefa semelhante a de julgamento de frases da bateria AWMA de memória de trabalho (Alloway, 2007). Pelos mesmos motivos já mencionados, de não estender $\mathrm{O}$ tempo de aplicação, decidiu-se que este pode ser incluído como subteste complementar, e não integrado a este instrumento.

Em função de os juízes julgarem a tarefa de cancelamento de figuras fácil, ela sofreu nova modificação, tornando-a semelhante à tarefa Bells Test (Gauthier, Dehaut \& Joanette, 1989), ou seja, ordem aleatória de apresentação das figuras e figuras em fundo preto, o que poderia aumentar a sensibilidade da tarefa. As demais sugestões em relação aos desenhos (modificações de detalhes) foram todas acatadas.

\section{Resultados da Etapa 5}

Essa etapa envolveu a aplicação de todas as tarefas após a reformulação total do instrumento, análise de tipos de erros (análises qualiquantitativas) e do desempenho das tarefas cuja pontuação é menos objetiva, como o processamento de inferências e as praxias construtivas. Os limites de tempo das tarefas que incluem esta medição em suas pontuações também foram definidos melhor após os estudos piloto.

As versões finais das tarefas de cancelamento de figuras (atenção) e go-no go mostraram-se sensíveis e suas instruções foram compreendidas pelas crianças. Os estudos piloto foram essenciais para análise das possíveis variações de respostas conforme a região (RS e SP), o que não ocorreu de forma substancial.

Uma modificação importante aqui observada foi quanto à sequência de aplicação das tarefas: na versão inicial a tarefa de memória episódica verbal (recordação de lista de palavras) era posterior às provas de linguagem. $\mathrm{Na}$ versão final do instrumento, a tarefa de recordação da lista de palavras (imediata) é a primeira prova a ser apresentada, evitando assim interferência das demais tarefas com estímulos lexicais. Entre a evocação imediata e a tardia, são apresentadas tarefas visuais também para evitar interferência.

\section{Discussão}

O presente estudo descreve o desenvolvimento do Instrumento de Avaliação Neuropsicológica Infantil Breve - NEUPSILIN-INF para crianças de 6 a 12 anos de idade, considerando características sociais, culturais e linguísticas da população brasileira, assim como particularidades do desenvolvimento infantil. De um modo geral, o processo transcorreu com critério e rigor, atendendo à recomendação da literatura internacional (por exemplo, Acevedo e colaboradores, 2009; Judd e colaboradores, 2009) no que concerne às etapas sucessivas e método apropriado de forma a otimizar a confiabilidade e as validades de um instrumento. A versão final do 
instrumento, apesar de manter o objetivo principal do NEUPSILIN de delinear um perfil neuropsicológico breve, traz tarefas mais adequadas ao exame neuropsicológico na segunda infância. Além da avaliação quantitativa, o NEUPSILIN-INF pode fornecer, em termos qualitativos, indícios de uso de estratégias cognitivas (análise dos tipos de erros).

Todas as etapas foram muito importantes, destacando-se os estudos piloto por se tratar do momento de verificação em contexto real de todos os materiais que compõem o NEUPSILIN-INF, contribuindo para identificar provas pouco sensíveis, estimar o tempo de aplicação total do instrumento, verificar a compreensibilidade de instruções e estímulos, assim como aprimorar o entendimento das normas de pontuação por parte dos examinadores. A primeira etapa foi caracterizada por um longo processo de análise, que demandou consultas à literatura nacional e internacional, bem como amplas discussões pela equipe sobre as formas de instrução e verificação do desempenho de cada subteste. Conforme destaca Pasquali (2001), no processo de validade de conteúdo de um instrumento, é preciso que se façam especificações que comportam três grandes temas: a) definição do conteúdo; b) explicitação dos processos cognitivos a serem avaliados; c) determinação da proporção relativa de representação no teste de cada tópico do conteúdo. No caso do NEUPSILIN-INF, esse processo resultou em um aporte de tarefas com fundamentação teórica considerando os achados mais recentes acerca da neuropsicologia do desenvolvimento (Michel, 2001) e da neuropsicologia cognitiva para cada processo examinado.

$\mathrm{Na}$ etapa de construção da primeira versão do instrumento, foi necessário o desenvolvimento de tarefas novas, especialmente ao se considerarem os possíveis diagnósticos neurológicos e neuropsiquiátricos em amostras de crianças, e as queixas mais frequentes de déficits neuropsicológicos na infância. Foram inseridas tarefas de processamento fonológico da linguagem, como de consciência fonológica (uma habilidade metalinguística) e de memória de trabalho (ou operacional) fonológica (repetição de pseudopalavras), cujos déficits são considerados subjacentes às dificuldades de leitura (Salles \& Parente, 2008, 2009; Vellutino, Fletcher, Snowling \& Scanlon, 2004; Frith, 1997). Considerandose a possibilidade de detecção de sintomas dos transtornos globais do desenvolvimento, acrescentouse uma tarefa de processamento de emoções em faces que, junto à de processamento de inferências, pode ser sensível a esse diagnóstico (Mercadante, Van der Gaag \& Schwartzman, 2006). Ainda há necessidade de mais estudos sobre a validade clínica dos testes de processamento de emoções faciais, apesar de grande importância em pesquisa. A tarefa de funções executivas go-no-go foi inserida por tratar-se de um conhecido paradigma de inibição e ser frequentemente citada na literatura como sensível a quadros como o transtorno do déficit de atenção/hiperativadade (Brocki \& Bohlin, 2004).

A análise de juízes especialistas confirmou que cada tarefa e funções contempladas no instrumento são essenciais para alcançar o objetivo proposto: ser um instrumento de rápida aplicação e com baixo custo, capaz de avaliar o desempenho neuropsicológico em crianças de 6 a 12 anos de idade (em idade escolar), com desenvolvimento típico e com possíveis transtornos do desenvolvimento. Conforme estabelece a literatura (Byrd e colaboradores, 2008, Hambleton \& Bollwark, 1991), o papel do Comitê de Especialistas (Expert Committee) é consolidar a versão apresentada, assim como auxiliar a desenvolver o que seria a versão pré-final do instrumento. Dessa forma, obteve-se uma das primeiras evidências de validade de conteúdo do NEUPSILIN-INF.

Considerando que a principal característica do NEUPSILIN-INF é delinear um perfil cognitivo breve de crianças, não foi possível contemplar a avaliação de todos os componentes cognitivos, tendo sido mantidos aqueles considerados essenciais para um exame inicial em sessão única. Para complementar as hipóteses diagnósticas funcionais iniciais possibilitadas pelo NEUPSILIN-INF, recomenda-se o uso de outros instrumentos (baterias e tarefas neuropsicológicas específicas) e procedimentos para investigação mais aprofundada.

Como se privilegiou o tempo de aplicação em uma sessão, algumas das limitações do presente instrumento são o número reduzido de itens por tarefa (em algumas funções avaliadas) e a pouca representatividade dos diferentes componentes das funções de atenção (apenas atenção focalizada), habilidades aritméticas (operações simples) e funções executivas (fluência verbal e go-no go). Salienta-se também que não puderam ser incluídas tarefas de processamento textual (nem compreensivo nem expressivo) e componentes mnemônicos implícitos.

Mesmo em face dessas limitações, o desenvolvimento criterioso desta ferramenta de investigação neuropsicológica para crianças deve contribuir para suprir, pelo menos parcialmente, a escassez de ferramentas nacionais padronizadas de avaliação breve dos processos neurocognitivos nesta faixa de desenvolvimento. Além disso, os procedimentos metodológicos aqui utilizados podem auxiliar no processo de desenvolvimento de outros instrumentos. Com os próximos estudos serão obtidas 
evidências de fidedignidade, validade de construto e de critério e dados normativos por subteste, considerando-se idade, escolaridade e tipo de escola, além de perfis neuropsicológicos clínicos de crianças com quadros neurológicos e/ou psiquiátricos.

\section{Referências}

Acevedo, A, Krueger, K. R., Navarro, E., Ortiz, F., Manly, J. J., Padilla-Ve'lez, M. M., Weintraub, S., López, O. L. \& Mungas, D. (2009). The Spanish translation and adaptation of the uniform data set of the National Institute on Aging Alzheimer's Disease Centers. Alz̧heimer Disease and Association Disorders, 23(2), 102-109.

Alloway, T P. (2007). Automated Working Memory Assessment: manual. Londres: Pearson.

Alloway, T. P., Gathercole, S. E., Willis, C. \& Adams, A. (2004). A structural analysis of working memory and related cognitive skills in young children. Journal of Experimental Child Psychology, 87, 85-106.

Brocki, K. C. \& Bohlin, G. (2004). Executive functions in children aged 6 to 13: a dimensional and developmental study. Developmental Neuropsychology, 26(2), 571-93.

Byrd, D., Arentoft, A., Scheiner, D., Westerveld, M. \& Baron, I. S. (2008). State of multicultural neuropsychological assessment in children: current research issues. Neuropsychological Review, 18, 214222.

Carey, P. F. \& Konkol, R. J. (2001). Neuropsychology: adaptation for a busy pediatric neurology clinic in a managed care setting. Paper presented at the meeting of the Child Neurology Society, Victoria, BC, Canada.

Cohen, M. J. (1997). Manual for the Children's Memory Scale. San Antonio: The Psychological Corporation.

Fagundes, A. J. F. M. (1985). Descrição, definição e registro do comportamento. São Paulo: Edicon.

Fonseca, R. P., Salles, J. F. \& Parente, M. A. M. P. (2008). Development and content validity of the Brazilian Brief Neuropsychological Assessment Battery NEUPSILIN. Psychology \& Neuroscience, 1, 55-62.

Fonseca, R. P., Salles, J. F. \& Parente, M. A. M. (2009). Instrumento de Avaliação Neuropsicológica Breve NEUPSILIN. São Paulo, SP: Vetor.
Frith, U. (1997). Brain, mind and behaviour in dyslexia. Em C. Hulme \& M. Snowling (Orgs.). Dyslexia: Biology, cognition and intervention. (pp. 1-19). California: Singular Publishing Group, INC.

Gathercole, S. (1998). The development of memory. Journal of Children Psychiatric, 39, 3-27.

Gauthier, L., Dehaut, F., \& Joanette, Y. (1989) The Bells Test: a quantitative and qualitative test for visual neglect. International Journal of Clinical Neuropsychology, 11, 49-54.

Hambleton, R. K. \& Bollwark, J. (1991). Adapting tests for use in different cultures: technical issues and methods. ITC Bulletin/ Bulletin CIT: International Test Bulletin Issues, 32(33), 3-32.

Hogan, T. P. (2006). Introdução à pratica de testes psicológicos. Rio de Janeiro: LTC.

Judd, T., Capetillo, D., Carrion-Baralt, J., Marmol, L. M., Miguel-Montes, L. S., Navarrete, M. G., Puente, A. E., Romero, H. R. \& Valdes, J. (2009). Professional considerations for improving the neuropsychological evaluation of Hispanics: a National Academy of Neuropsychology education paper. Archives of Clinical Neuropsychology, 24, 127135.

Laurent-Vannier, A., Chevignard, M., Pradat-Diehl, P., Abada, G. \& De Agostini, M. (2006). Assessment of unilateral spatial neglect in children using the Teddy Bear Cancellation Test. Developmental Medicine \& Child Neurology, 48, 120-125.

Lezak, M., Howieson, D. \& Loring, D. (2004). Neuropsychological assessment. Nova Iorque: Oxford University Press.

Matute E., Rosselli M., Ardila A. \& Ostrosky-Solis F. (2007). Evaluación neuropsicológica infantil. México: Universidad de Guadalajara.

Mercadante, M. T., Van Der Gaag, R. J. \& Schwartzman, J. S. (2006). Transtornos invasivos do desenvolvimento não-autísticos: síndrome de Rett, transtorno desintegrativo da infância e transtornos invasivos do desenvolvimento sem outra especificação. Revista Brasileira de Psiquiatria, 28(1), 12-20.

Michel, G. F. (2001). A developmentalpsychobiological approach to developmental neuropsychology. Developmental Neuropsychology, 19, 11-32.

Miranda, M. C. (2000) Recordação livre de palavras e figuras em crianças pré-escolares. (Dissertação de Mestrado). 
São Paulo: Universidade Federal de São Paulo Escola Paulista de Medicina.

Miranda, M. C. (2006). Avaliação neuropsicológica quantitativa e qualitativa: ultrapassando os limites da psicometria. Em C. B. Mello, M. C. Miranda \& M. Muszkat. (Orgs.). Neuropsicologia do desenvolvimento. Conceitos e abordagens. (pp. 127-143). São Paulo: Memnon.

Pasquali, L. (2001). Técnicas de Exame Psicológico - TEP. Vol. I: Fundamentos das técnicas psicológicas. São Paulo: Casa do Psicólogo.

Piccolo, L. R. (2010). Relaçoes entre variáveis psicossociais e cognitivas e o desempenho em leitura em crianças de uma coorte populacional. (Dissertação de Mestrado). Porto Alegre: Universidade Federal do Rio Grande do Sul.

Pompéia, S., Miranda, M. C. \& Bueno, O. F. A. (2001). A set of 400 pictures standardized for Portuguese: norms for name agreement, familiarity and visual complexity for children and adults. Arquivos de Neuro-Psiquiatria, 59, 330-337.

Reitan, R. M. \& Wolfson, D. (2004a). The trail making test as an initial screening procedure for neuropsychological impairment in older children. Archives of Clinical Neuropsychology, 19, 281-288.

Salles, J. F. \& Parente, M. A. M. P. (2008). Variabilidade no desempenho em tarefas neuropsicológicas entre crianças de $2^{\mathrm{a}}$ série com dificuldades de leitura e escrita. Arquivos Brasileiros de Psicologia, 60(1), 32-44.
Salles, J. F. \& Parente, M. A. P. (2009). As dificuldades de leitura e escrita em crianças/adolescentes: abordagem neuropsicológica cognitiva. Em V. G. Haase, F. O. Ferreira \& F. J. Penna (Orgs.). Aspectos biopsicossociais da saúde na infância $e$ adolescência (pp. 353-373). Belo Horizonte: COOPMED.

Salles, J. F., Fonseca, R. P., Miranda, M. C., Mello C. B., Cruz-Rodrigues, C. \& Barbosa, T. (no prelo). Instrumento de Avaliação Neuropsicológica Breve Infantil. São Paulo: Vetor.

Simões, M. R. (2002). Avaliação neuropsicológica em crianças e adolescentes. Em R. Primi (Org.). Temas em avaliação psicológica (pp. 26-34). Campinas: Instituto Brasileiro de Avaliação Psicológica.

Strauss, E., Sherman, E. M. S. \& Spreen, O. (2006). A compendium of neuropsychological tests: administration, norms and commentary ( $3^{\mathrm{a}} \mathrm{ed}$.). Nova Iorque: Oxford University Press.

Vellutino, F. R., Fletcher J. M., Snowling M. J \& Scanlon D. M. (2004). Specific reading disability (dyslexia): what have we learned in the past four decades? Journal of Child Psychology and Psychiatry, 45(1), 2-40.

Recebido em 15/07/2011

Reformulado em 02/09/2011

Aprovado em 25/10/2011

Os autores agradecem ao fomento de pesquisa desta editora e ao do CNPq (processo número 400787/2007-5)

Sobre as autoras:

Jerusa Fumagalli de Salles é fonoaudióloga, mestre e doutora em psicologia, profa. adjunta do Instituto de Psicologia, Programa de Pós-Graduação em Psicologia, Universidade Federal do Rio Grande do Sul - UFRGS, coordenadora do Núcleo de Estudos em Neuropsicologia Cognitiva (Neurocog): www.ufrgs.br/neuropsicologia.

Rochele Paz Fonseca é professora adjunta da Faculdade de Psicologia e do Programa em Pós-Graduação em Psicologia (Cognição Humana), coordenadora do Grupo Neuropsicologia Clínica e Experimental (GNCE), PUCRS. Possui PósDoutoramento em Ciências Biomédicas pela Universidade de Montreal, em Neurorradiologia (UFRJ) e em Psicologia Clínica e Neurociências (PUC-Rio) e Doutorado em Psicologia (UFRGS, Universidade de Montreal).

Camila Cruz-Rodrigues é psicóloga, mestre e doutora em Ciências pela UNIFESP, coordenadora do Ambulatório de Distúrbios de Aprendizagem do NANI e professora do Curso de Psicologia, Centro de Ciências Biológicas e da Saúde da Universidade Presbiteriana Mackenzie.

Claudia Berlim de Mello é psicóloga, doutora em Psicologia pela USP e coordenadora do NANI.

Thais Barbosa é fonoaudióloga, mestre e doutoranda em Ciências pela UNIFESP, coordenadora do Ambulatório de Distúrbios de Aprendizagem do NANI.

Mônica C Miranda é psicóloga, doutora em Ciências, coordenadora do NANI e orientadora da UNIFESP. 
